\title{
Randomized Open Label Phase III Trial of Irinotecan Plus Capecitabine versus Capecitabine Monotherapy in Patients with Metastatic Breast Cancer Previously Treated with Anthracycline and Taxane: PROCEED Trial (KCSG BR 11-01)
}

\section{In Hae Park, MD ${ }^{1}$ \\ Seock-Ah Im, MD² \\ Kyung Hae Jung, $M D^{3}$ \\ Joo Hyuk Sohn, MD ${ }^{4}$ \\ Yeon Hee Park, MD \\ Keun Seok Lee, MD 1 \\ Sung Hoon Sim, MD \\ Kyong-Hwa Park, $\mathrm{MD}^{6}$ \\ Jee Hyun Kim, MD \\ Byung Ho Nam, PhD 8 ,a \\ Hee-Jun Kim, MD9 \\ Tae-Yong Kim, MD2 \\ Kyung-Hun Lee, MD2 \\ Sung-Bae Kim, MD ${ }^{3}$ \\ Jin-Hee Ahn, MD \\ Suee Lee, MD ${ }^{10}$ \\ Jungsil Ro, MD}

*A list author's affiliations appears at the end of the paper.

\section{Purpose}

We investigated whether irinotecan plus capecitabine improved progression-free survival (PFS) compared with capecitabine alone in patients with human epidermal growth factor 2 (HER2) negative and anthracycline and taxane pretreated metastatic breast cancer (MBC).

\section{Materials and Methods}

A total of 221 patients were randomly assigned to irinotecan $\left(80 \mathrm{mg} / \mathrm{m}^{2}\right.$, days 1 and 8$)$ and capecitabine $\left(1,000 \mathrm{mg} / \mathrm{m}^{2}\right.$ twice a day, days $\left.1-14\right)$ or capecitabine alone $\left(1,250 \mathrm{mg} / \mathrm{m}^{2}\right.$ twice a day, days 1-14) every 3 weeks. The primary endpoint was PFS.

\section{Results}

There was no significant difference in PFS between the combination and monotherapy arm (median, 6.4 months vs. 4.7 months; hazard ratio [HR], 0.84; 95\% confidence interval [Cl], 0.63 to 1.11; $p=0.84$ ). In patients with triple-negative breast cancer (TNBC, $n=90$ ), the combination significantly improved PFS (median, 4.7 months vs. 2.5 months; HR, 0.58; 95\% $\mathrm{Cl}, 0.37$ to $0.91 ; \mathrm{p}=0.02$ ). Objective response rate was numerically higher in the combination arm, though it failed to reach statistical significance ( $44.4 \%$ vs. $33.3 \%, p=0.30)$. Overall survival did not differ between arms (median, 20.4 months vs. 24.0 months; $p=0.63$ ). While grade 3 or 4 neutropenia was more common in the combination arm (39.6\% vs. 9.0\%), hand-foot syndrome was more often observed in capecitabine arm. Quality of life measurements in global health status was similar. However, patients in the combination arm showed significantly worse symptom scales especially in nausea/vomiting and diarrhea.

\section{Conclusion}

Irinotecan plus capecitabine did not prove clinically superior to single-agent capecitabine in anthracycline- and taxane-pretreated HER2 negative MBC patients. Toxicity profiles of the two groups differed but were manageable. The role of added irinotecan in patients with TNBC remains to be elucidated.

\section{Key words}

Metastatic breast cancer, Irinotecan, Capecitabine, Clinical trial, Progression-free survival
Department of Cancer Biomedical Science, National Cancer Center Graduated School of Cancer Science and Policy, National Cancer Center, 323 Ilsan-ro, Ilsandong-gu, Goyang 10408, Korea

Tel: 82-31-920-1680

Fax: 82-31-920-0858

E-mail: jungsro@ncc.re.kr

Received November 27, 2017

Accepted February 12, 2018

Published Online February 14, 2018

aPresent address: The Institute of Advanced Clinical \& Biomedical Research,

Herings, Seoul, Korea 


\section{Introduction}

Despite substantial progress in patient management over the past few decades, metastatic breast cancer (MBC) is still considered incurable. To date, the mainstay of treatment for $\mathrm{MBC}$ is cytotoxic chemotherapy which has been proved to prolong survival and improve quality of life (QoL) in patients with MBC [1]. Nevertheless, there are few alternatives if patients show resistance to anthracycline- or taxanebased regimens. Therefore, new treatment options are urgently needed, particularly for patients with heavily pretreated refractory disease.

Irinotecan is a topoisomerase I (Top1) inhibitory pro-drug that is enzymatically converted to the active metabolite SN38 [2]. It preserves single-strand DNA breaks and binds to Top1-DNA complexes during DNA replication and transcription resulting in double-stranded DNA breaks and eventual cell death [3]. Irinotecan has been used in conjunction with other chemotherapeutic agents to treat colorectal, lung, and gastric cancers $[4,5]$. Specifically, irinotecan combined with capecitabine or 5-fluorouracil has been widely accepted as the first line therapy in metastatic colorectal cancer, given its synergistic effects $[4,6]$. In breast cancer, its antitumor activity has been assessed either in single-agent use or in combination with other therapeutics. As monotherapy, objective response rates (ORRs) have ranged from 14\%-23\%, and median response duration was reported around 4.5 months in patients with heavily pretreated MBC [7]. Meanwhile, irinotecan when added to docetaxel, gemcitabine, or cisplatin showed response rates of $31 \%-64 \%$ and median time-to-progression of 6.7-8 months in patients with MBC $[8,9]$. We previously conducted a phase II single-arm study investigating the feasibility of using irinotecan plus capecitabine (IX) in patients with anthracycline- and taxanepretreated MBC. As a result, ORR was 58.3\% (95\% confidence interval [CI], 42.2 to 72.9 ), and median progressionfree survival (PFS) was 7.6 months (95\% CI, 5.0 to 10.2) [10]. Toxicities of this combination were manageable and tolerable: neutropenia $\geq$ grade $3,58.4 \%$; febrile neutropenia $5.6 \%$; and grade 3 diarrhea, $2.8 \%$. Based on these promising results, a randomized, multicenter, and open-label phase III clinical trial was conducted to determine whether irinotecan plus capecitabine was superior to capecitabine alone in terms of PFS for patients with anthracycline- and taxane-pretreated MBC.

\section{Materials and Methods}

\section{Study design and patients}

This open-label, multicenter, randomized phase III study (PROCEED; Randomized Phase III trial of irinotecan plus capecitabine versus capecitabine monotherapy in patients with MBC previously treated with anthracycline and taxane) was conducted at 10 sites in South Korea. Eligible patients were $\geq 20$ years old, with Eastern Cooperative Oncology Group (ECOG) performance status scores of 0 or 1 and human epidermal growth factor 2 (HER2)-negative MBC. All participants had previously received anthracycline- and taxane-based chemotherapies and no more than two previous cytotoxic regimens for their metastatic disease. Patients who experienced disease recurrence within 1 year after completion of neo/adjuvant anthracycline- and taxane-based chemotherapy were also eligible without any treatment for their metastatic disease. Other inclusion criteria were as follows: (1) resolution of treatment-related toxicities to $\leq$ grade 1, according to National Cancer Institute Common Terminology Criteria for Adverse Events (NCI-CTCAE) ver. 3.0; (2) adequate organ function; and (3) negative serum pregnancy test. The main exclusion criteria were symptomatic brain metastasis, uncontrolled infection, or serious medical problems such as heart failure, uncontrolled diabetes, or gastrointestinal problems with absorption.

Patients were evenly randomized to either the irinotecan/ capecitabine combination (IX) or the single-agent capecitabine $(X)$ study arm. Randomization was stratified by hormone receptor status and presence of visceral metastasis. The primary study endpoint was PFS in the intention-to-treat (ITT) population, with secondary endpoints of ORR, overall survival (OS), and safety.

\section{Study treatment and assessment}

Irinotecan was administered intravenously at a dose of 80 $\mathrm{mg} / \mathrm{m}^{2}$ over 90 minutes on days 1 and 8 , and capecitabine was given per oral as $1,000 \mathrm{mg} / \mathrm{m}^{2}$ dose twice daily on days $1-14$ every 3 weeks. In the single-agent capecitabine arm, the dosage was $1,250 \mathrm{mg} / \mathrm{m}^{2}$ twice daily on days 1-14 every 3 weeks. To lessen irinotecan-induced toxicities, we concomitantly administered urodeoxycholinic acid (200 $\mathrm{mg}$ three times), sodium bicarbonate (1,000 mg twice), and magnesium oxide (300 mg) for 3 days. Loperamide was used to manage delayed diarrhea. Dose modifications were performed for patients with toxicities with the same manner as that utilized in the previous phase II clinical trial [10]. Capecitabine interruptions or adjustments to manage toxicities were permitted at the physician's discretion. Treatment was continued until 
disease progression, unacceptable toxicities, or patient request for discontinuation.

To assess tumors, the Response Evaluation Criteria in Solid Tumors (RECIST) ver. 1.1 was applied at screening and every 6 weeks after treatment initiation. Adverse events (AEs) were evaluated and recorded at baseline and throughout treatment using the NCI-CTCAE ver. 3.0. The European Organization for Research and Treatment of Cancer (EORTC) Quality of Life Core Questionnaire (QLQ-C30) was also administered to each patient at baseline and after every two cycles of chemotherapy [11]. For this study, we used the Korean version of QLQ-C30 which has been validated [12].

\section{Statistical analysis}

Prior data had provided a median PFS value for the control arm of capecitabine monotherapy $(\mathrm{X})$, which was 4.0 months in a similar patient population [13,14]; and we anticipated a median PFS of 6.0 months for combined irinotecan/capecitabine treatment (IX). Patients would be randomized to each group at a 1:1 ratio, necessitating a total enrollment of 200 patients for a power of $80 \%$. The type I error probability associated with testing this null hypothesis was 0.05. Assuming a withdrawal rate of $10 \%$, a total patient accrual of 222 was warranted.

All patients participating in randomization were included in the ITT population, which served for all survival analysis. Safety was assessed in those receiving at least one dose of a study treatment regimen, and ORR was evaluated in patients with measurable disease. PFS and OS estimates were generated by Kaplan-Meier method and compared via log-rank test. The Cox proportional hazards analysis was applied to weigh outcomes of the experimental treatment against those of the control arm, examining variables that might impact treatment response. ORR was calculated as the proportion of patients with complete or partial tumor responses.

The QLQ-C30 data were linearly transformed to yield scores from 0 to 100 , according to the EORTC scoring manual [11]. The mean change in QoL score from baseline was calculated separately, and analysis of variance (ANOVA) with repeated measures was used for between-group comparison of mean change in QoL score. All tests were 2-sided, and we considered p-value of $<0.05$ as statistically significant. All statistics were calculated using SPSS ver. 21.0 software (IBM Corp., Armonk, NY).

\section{Ethical statement}

This trial complied fully with guidelines for Good Clinical Practice and the Declaration of Helsinki. Written informed consent was obtained from each patient. Approval of the protocol and of any amendments was obtained from an independent ethics committee for each site. This study was registered with ClinicalTrials.gov (number NCT01501669).

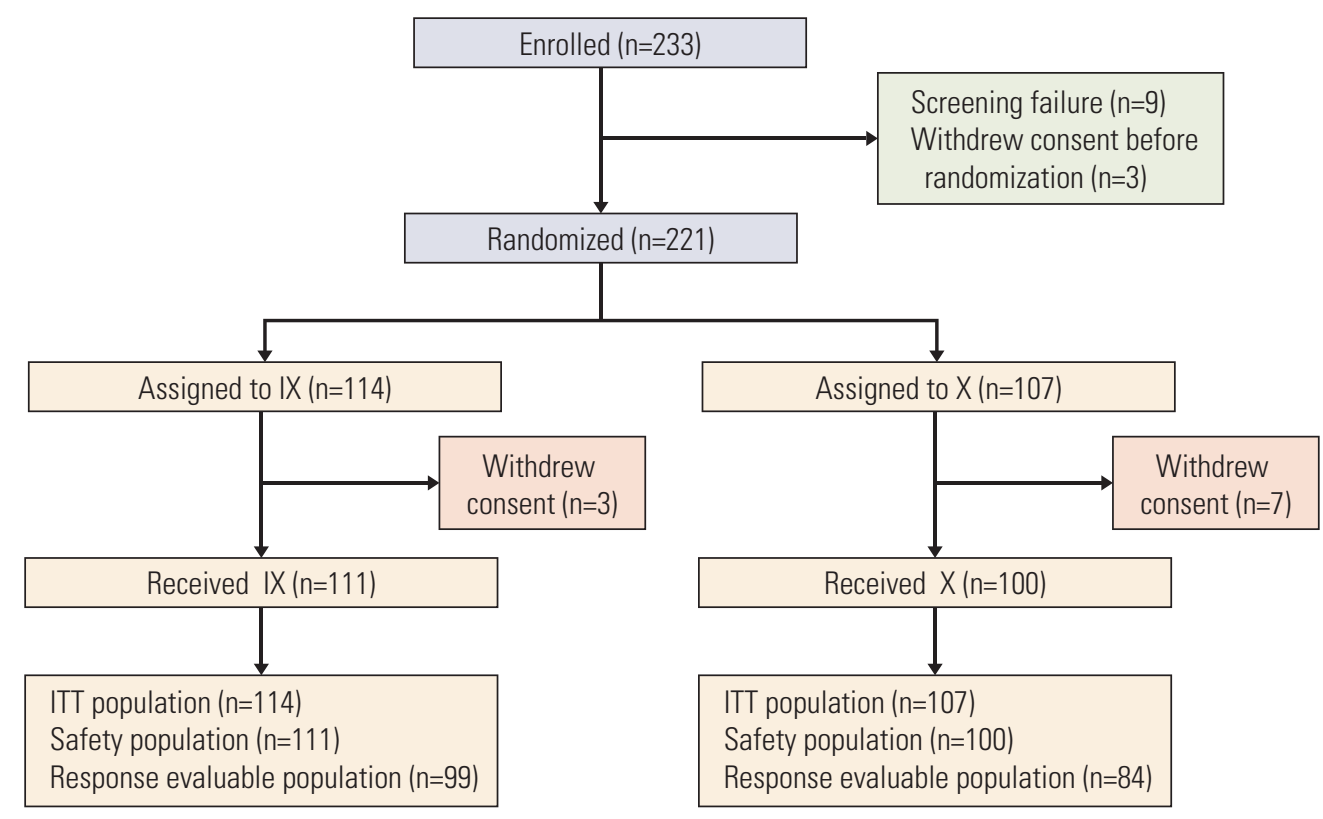

Fig. 1. Study flowchart. ITT, intention-to-treat. 
Table 1. The baseline patient characteristics

\begin{tabular}{|c|c|c|c|}
\hline Characteristic & IX ( $n=114)$ & $X(n=107)$ & p-value \\
\hline Age, median (range, yr) & $50(29-73)$ & $49(30-80)$ & 0.64 \\
\hline \multicolumn{4}{|l|}{ ECOG PS } \\
\hline 0 & $26(22.8)$ & $24(22.4)$ & 0.82 \\
\hline 1 & $87(76.3)$ & $81(75.7)$ & \\
\hline 2 & $1(0.9)$ & $2(1.9)$ & \\
\hline \multicolumn{4}{|l|}{ Menopausal status } \\
\hline Pre-menopause & $30(26.3)$ & $32(29.9)$ & 0.65 \\
\hline Post-menopause & $84(73.7)$ & $75(70.1)$ & \\
\hline \multicolumn{4}{|l|}{ ER/PgR } \\
\hline Positive & $62(54.4)$ & $69(64.5)$ & 0.13 \\
\hline Negative & $52(45.6)$ & $38(35.5)$ & \\
\hline (Neo)Adjuvant chemotherapy & $89(78.1)$ & $77(72.0)$ & 0.35 \\
\hline Adjuvant endocrine & $48(42.1)$ & $43(40.2)$ & 0.79 \\
\hline \multicolumn{4}{|l|}{ Visceral metastasis } \\
\hline Yes & $66(57.9)$ & $63(58.9)$ & 0.89 \\
\hline No & $48(42.1)$ & $44(41.1)$ & \\
\hline \multicolumn{4}{|c|}{ Previous palliative endocrine therapy } \\
\hline Yes & $38(33.3)$ & $44(41.1)$ & 0.23 \\
\hline No & $76(66.7)$ & $63(58.9)$ & \\
\hline \multicolumn{4}{|l|}{ No. of previous chemotherapies } \\
\hline 0 & $13(11.4)$ & $13(12.1)$ & 0.27 \\
\hline 1 & $61(53.5)$ & $48(44.9)$ & \\
\hline$\geq 2$ & $40(35.1)$ & $46(43.0)$ & \\
\hline
\end{tabular}

Values are presented as number (\%). IX, irinotecan and capecitabine combination; X, capecitabine alone; ECOG PS, Eastern Cooperative Oncology Group Performance Score; ER, estrogen receptor; PgR, progesterone receptor.

\section{Results}

\section{Baseline characteristics and study treatment}

Between March 2011 and May 2016, 233 patients were enrolled. Of these, 114 patients were randomized to arm IX and 107 to arm X (Fig. 1). Three patients in arm IX and seven patients in arm $X$ withdrew consent before proceeding to treatment. Consequently, 211 patients received at least one dose of assigned treatment and they were included in safety analysis. There were no statistically significant differences between groups in terms of age, performance status, and visceral metastasis. In arms IX and X, 54.4\% and $64.5 \%$ of patients, had hormonal receptor positive disease. Two-thirds of the patients received prior chemotherapy in the (neo) adjuvant setting. The number of patients given more than one line of chemotherapy for metastatic disease was 40 $(35.1 \%)$ in arm IX and 46 (43.0\%) in arm X (Table 1). Around $10 \%$ of patients received study regimens as the first-line therapy for MBC.

\section{Efficacy}

During a median follow-up period of 22.8 months (95\% CI, 18.8 to 26.8$)$, a total of 118 deaths had occurred, $62(54.4 \%)$ in arm IX and $56(52.3 \%)$ in arm X. The two arms showed no significant difference in median PFS (arm IX: 6.4 months; 95\% CI, 4.7 to 8.1 and arm X: 4.7 months; $95 \%$ CI, 3.7 to 5.7; $\mathrm{p}=0.21$ ) (Fig. 2A). Median OS in the two arms were also similar (IX: 20.4 months; 95\% CI, 16.6 to 24.2 and X: 24.0 months; 95\% CI, 17.1 to 30.9; $\mathrm{p}=0.63$ ) (Fig. 2B). Those patients ( $\mathrm{n}=183$ ) presenting with measurable disease at baseline were accessed for response analysis (Table 2). Objective responses were observed in 44 patients $(44.4 \%)$ of arm IX and 28 patients (33.3\%) of arm X arm, without significant difference $(\mathrm{p}=0.30)$ (Table 2).

In subgroup analysis, combination (IX) treatment showed significant improvement in PFS for patients with the triplenegative breast cancer (TNBC) subtype (hazard ratio [HR], 0.58; $95 \%$ CI, 0.37 to 0.91; $\mathrm{p}=0.02$ ) (Fig. 3). Median PFS of the TNBC subgroup in arm IX was 4.7 months (95\% CI, 3.5 to 5.9), compared with 2.5 months (95\% CI, 1.2 to 3.8) in arm $X$ $(\mathrm{p}=0.01)$ (Fig. 4A). However, such increase in PFS did not 
A

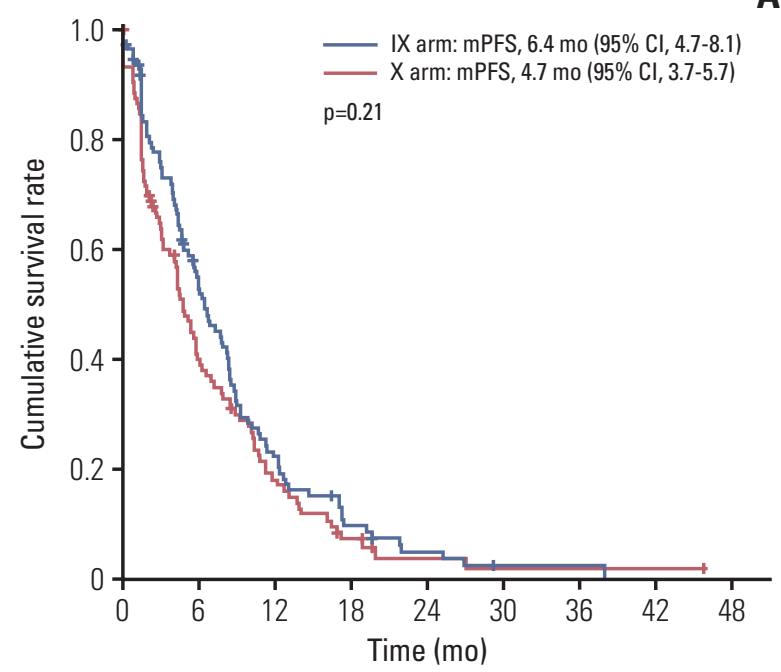

No. at risk

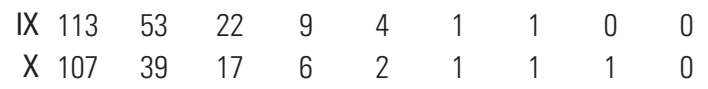

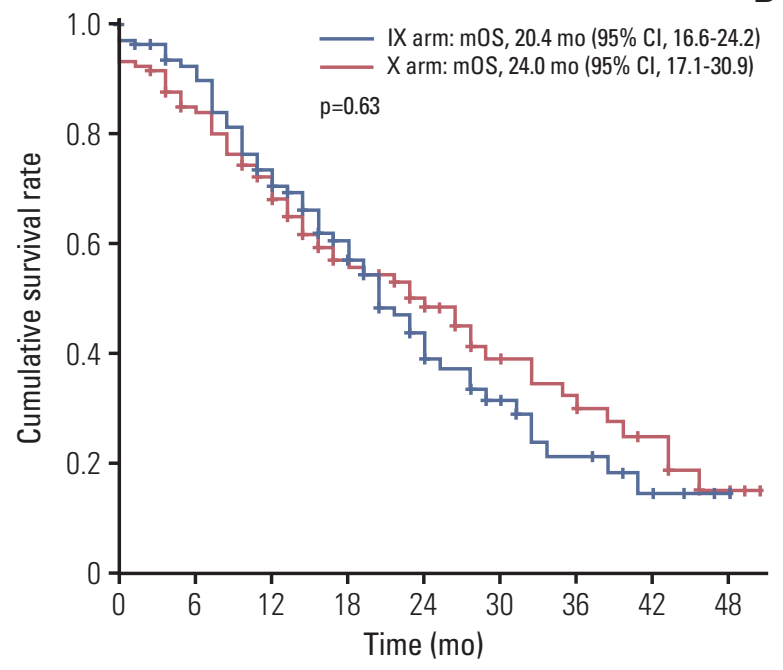

No. at risk

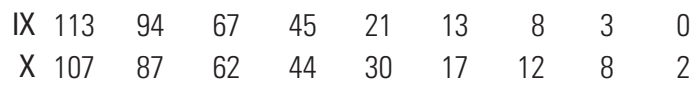

Fig. 2. Survival analysis in the intention-to-treat population. Kaplan-Meier curve for progression-free survival (PFS) (A) and overall survival (OS) (B) between irinotecan and capecitabine combination (IX) and capecitabine alone (X). mPFS, median PFS; mOS, median OS; CI, confidence interval.

Table 2. Tumor responses for patients with measurable disease

\begin{tabular}{lccc} 
& IX $(\mathbf{n = 9 9 )}$ & $\mathbf{X}(\mathbf{n}=84)$ & p-value \\
CR & $4(4.0)$ & $1(1.2)$ & \\
PR & $40(40.4)$ & $27(32.1)$ & \\
SD & $30(30.3)$ & $26(31.0)$ & \\
PD & $16(16.2)$ & $22(26.2)$ & \\
Not known & $9(9.1)$ & $8(9.5)$ & \\
ORR & $44(44.4)$ & $28(33.3)$ & 0.30 \\
\hline
\end{tabular}

Values are presented as number (\%). IX, irinotecan and capecitabine combination; $X$, capecitabine alone; $C R$, complete response; $\mathrm{PR}$, partial response; $\mathrm{SD}$, stable disease; $\mathrm{PD}$, progressive disease; ORR, objective response rate (CR+PR).

lead to the prolongation of OS in this subgroup (median OS, 18.0 months; $95 \%$ CI, 13.0 to 23.0 vs. 13.2 months; $95 \%$ CI, 6.7 to $19.7 ; \mathrm{p}=0.36$ ) (Fig. 4B).

\section{Safety and QoL data}

Patients who received at least one dose of study treatment were included in safety and QoL analysis. More patients in arm IX required dose reductions or interruptions (Table 3). The most common reasons for dose reduction or interruption in IX arm were hematologic toxicities $(85.2 \%, 71.1 \%)$ and hand-foot syndrome $(50.0 \%, 28.0 \%)$ in X arm. Permanent therapeutic discontinuation was rare: one patient in arm IX arm (arrhythmia) and three patients in $\operatorname{arm} \mathrm{X}$ ( $\mathrm{n}=1$, infection; $\mathrm{n}=2$, hematologic toxicities). In arm IX, the more common AEs included hematologic toxicities and diarrhea (Table 4). In particular, most of neutropenia and anemia occurred in IX arm were more than grade 2 which required dose modification of study drugs. Although neutropenia was the most common $\mathrm{AE}$, the frequency of neutropenic fever was quite low in IX arm ( $n=1,2.6 \%)$. On the other hand, hand-foot syndrome of any grade was more often manifested in arm X $(53.0 \%$ vs. $31.5 \%)$. None of the AEs in either treatment arm resulted in death.

QoL assessments were conducted at baseline and after every two cycles after randomization. However, data obtained after cycle 11 could not be reliably evaluated, with $<10 \%$ of patients remaining in the study beyond this time point. Significant differences in favor of arm $X$ were noted for diarrhea and nausea/ vomiting symptom scales $(p<0.05)$. The differences observed between treatment arms in other functional scales and in the global health scale were not significant (Fig. 5A and B). 


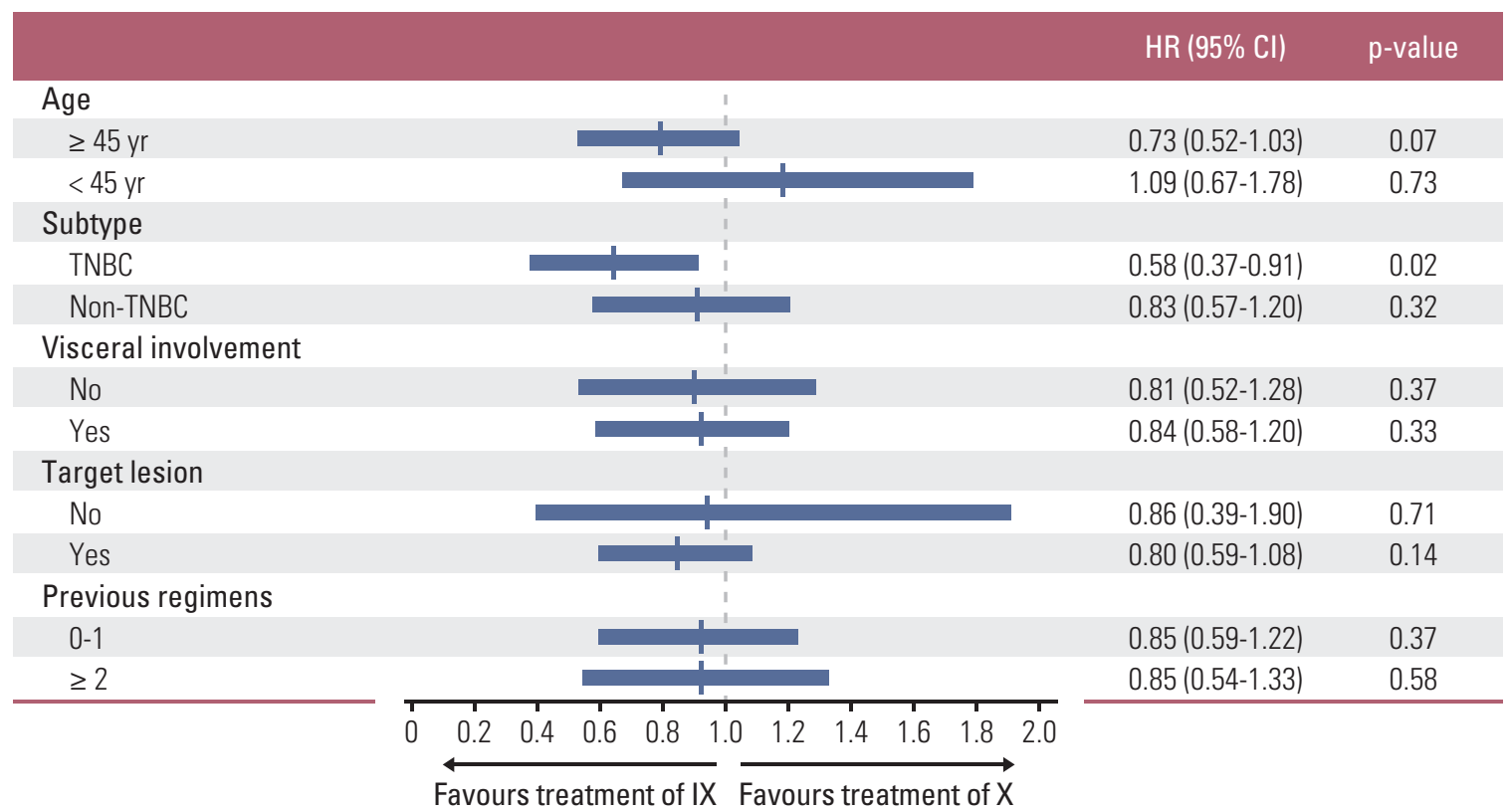

Fig. 3. The forest plots of progression-free survival in subgroups stratified by clinical factors. IX, irinotecan and capecitabine combination; $\mathrm{X}$, capecitabine; HR, hazard ratio; $\mathrm{CI}$, confidence interval; TNBC, triple negative breast cancer.

A
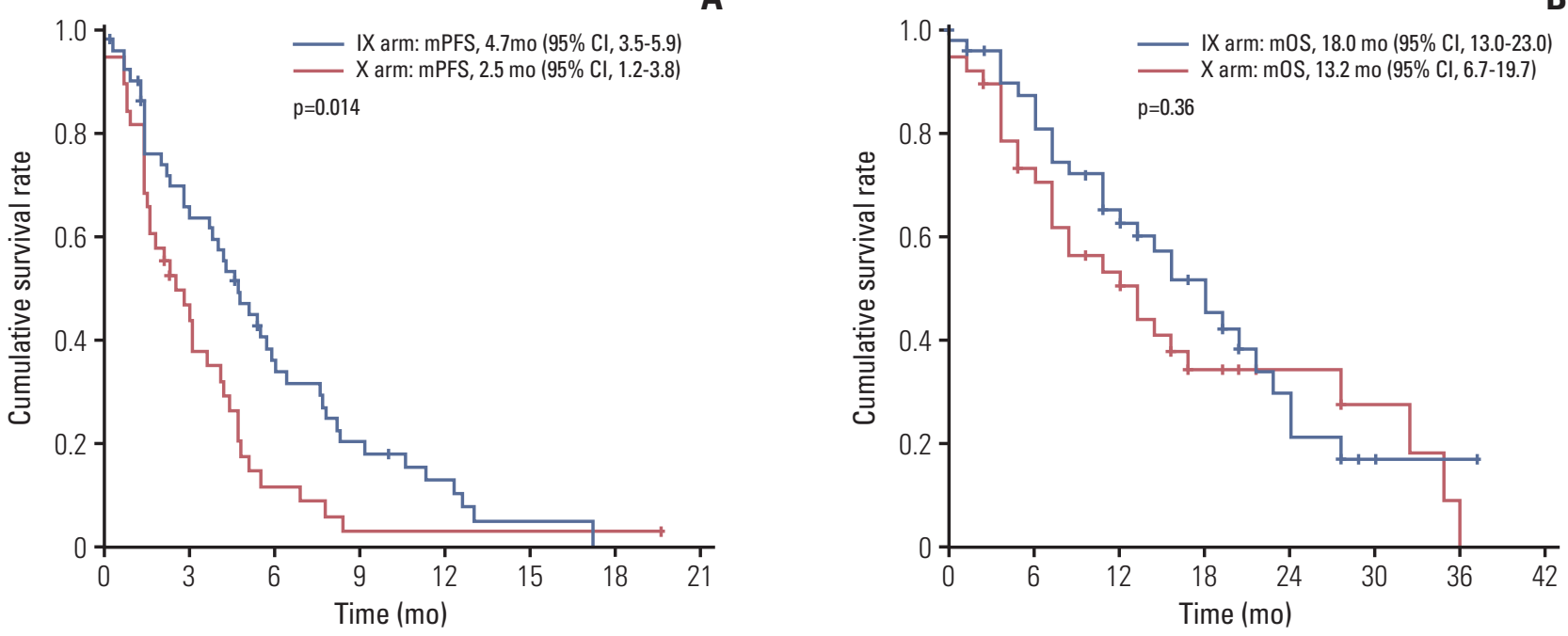

No. at risk

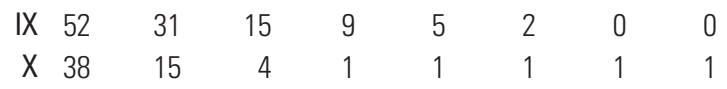

No. at risk

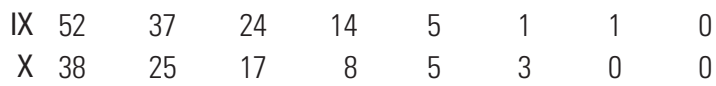

Fig. 4. Survival analysis in the triple negative breast cancer subgroup. Kaplan-Meier curve for progression-free survival (PFS) (A) and overall survival (OS) (B) between irinotecan and capecitabine combination (IX) and capecitabine alone (X). mPFS, median PFS; mOS, median OS; CI, confidence interval. 
Table 3. Dose modification of study drugs according to AEs

\begin{tabular}{|c|c|c|}
\hline & IX $(n=111)$ & $X(n=100)$ \\
\hline Dose reduction & $61(54.9)$ & $34(34.0)$ \\
\hline Hematologic AE & $52(85.2)$ & $9(26.5)$ \\
\hline Hand-foot syndrome & $12(23.1)$ & $17(50.0)$ \\
\hline Diarrhea & $4(6.6)$ & 0 \\
\hline Dose interruption & $38(34.2)$ & $25(25.0)$ \\
\hline Hematologic AE & $27(71.1)$ & $14(56.0)$ \\
\hline Hand-foot syndrome & $5(13.2)$ & $7(28.0)$ \\
\hline Neutropenic fever & $1(2.6)$ & $1(4.0)$ \\
\hline Diarrhea & $1(2.6)$ & $1(4.0)$ \\
\hline \multirow[t]{2}{*}{ Treatment discontinuation } & $1(0.9)$ & $3(3.0)$ \\
\hline & 1 (arrhythmia) & 1 (infection), 2 (hematologic AE) \\
\hline
\end{tabular}

Values are presented as number $(\%)$. AE, adverse events; IX, irinotecan and capecitabine combination; $\mathrm{X}$, capecitabine alone.

Table 4. Treatment related adverse events in both arms

\begin{tabular}{|c|c|c|c|c|c|}
\hline & \multicolumn{2}{|c|}{ IX $(n=111)$} & \multicolumn{2}{|c|}{$X(n=100)$} & \multirow{2}{*}{ p-value } \\
\hline & Grade 1-2 & Grade 3-4 & Grade 1-2 & Grade 3-4 & \\
\hline \multicolumn{6}{|l|}{ Hematologic AE } \\
\hline Neutropenia & $26(23.4)$ & $44(39.6)$ & $7(7.0)$ & $9(9.0)$ & $<0.001$ \\
\hline Anemia & $3(2.7)$ & $16(14.4)$ & $10(10.0)$ & $1(1.0)$ & $<0.001$ \\
\hline Thrombocytopenia & $7(6.3)$ & 0 & $4(4.0)$ & 0 & 0.45 \\
\hline \multicolumn{6}{|l|}{ Non-hematologic AE } \\
\hline Hand-foot syndrome & $33(29.7)$ & $2(1.8)$ & $49(49.0)$ & $4(4.0)$ & 0.007 \\
\hline Diarrhea & $46(41.4)$ & $3(2.7)$ & $29(29.0)$ & $1(1.0)$ & 0.012 \\
\hline Nausea/Vomiting & $61(54.9)$ & 0 & $36(36.0)$ & $2(2.0)$ & 0.03 \\
\hline Liver function abnormality & $2(1.8)$ & 0 & $7(7.0)$ & $1(1.0)$ & 0.098 \\
\hline Paronychia & $2(1.8)$ & 0 & $4(4.0)$ & $1(1.0)$ & 0.36 \\
\hline Edema & $9(8.1)$ & 0 & $5(5.0)$ & 0 & 0.37 \\
\hline Asthenia & $3(2.7)$ & 0 & $5(5.0)$ & 0 & 0.38 \\
\hline Insomnia & $15(13.5)$ & 0 & $7(7.0)$ & 0 & 0.12 \\
\hline
\end{tabular}

Values are presented as number (\%). IX, irinotecan plus capecitabine arm; $\mathrm{X}$, capecitabine alone arm; AE, adverse events.

\section{Discussion}

In general, combination therapy for MBC showed prolonged PFS with higher response rate compared to monotherapy, though such beneficial effects did not result in improved OS [15-18]. In this phase III study, the addition of irinotecan to capecitabine (IX) did not show the superior clinical efficacies to capecitabine monotherapy $(X)$ in patients with MBC previously exposed to anthracycline and taxane based treatment. The study was designed based on the assumption that combination treatment (IX) would prolong PFS 2.0 months beyond the 4.0-month PFS of monotherapy
(X). In our results, the PFS of arm IX was 6.4 months $(95 \%$ CI, 4.7 to 8.1), and that of arm X was 4.7 months (95\% CI, 3.7 to 5.7). For overall response rate, IX showed numerically higher response rate, however it did not reach statistical significance $(44.4 \%$ vs. $33.3 \%, \mathrm{p}=0.30)$. There are several possible explanations for the failure of this study. First, the number of patients with estrogen receptor- or progesterone receptor-positive disease in arm $X$ arm was higher $(64.5 \%$ vs. $54.4 \%, \mathrm{p}=0.13)$, though it was not statistically significant. Such imbalance between two groups may have had caused the better PFS of arm $X$ than expected. Second, the dose reductions and interruptions due to more frequent and severe hematologic toxicities of the combination treatment 

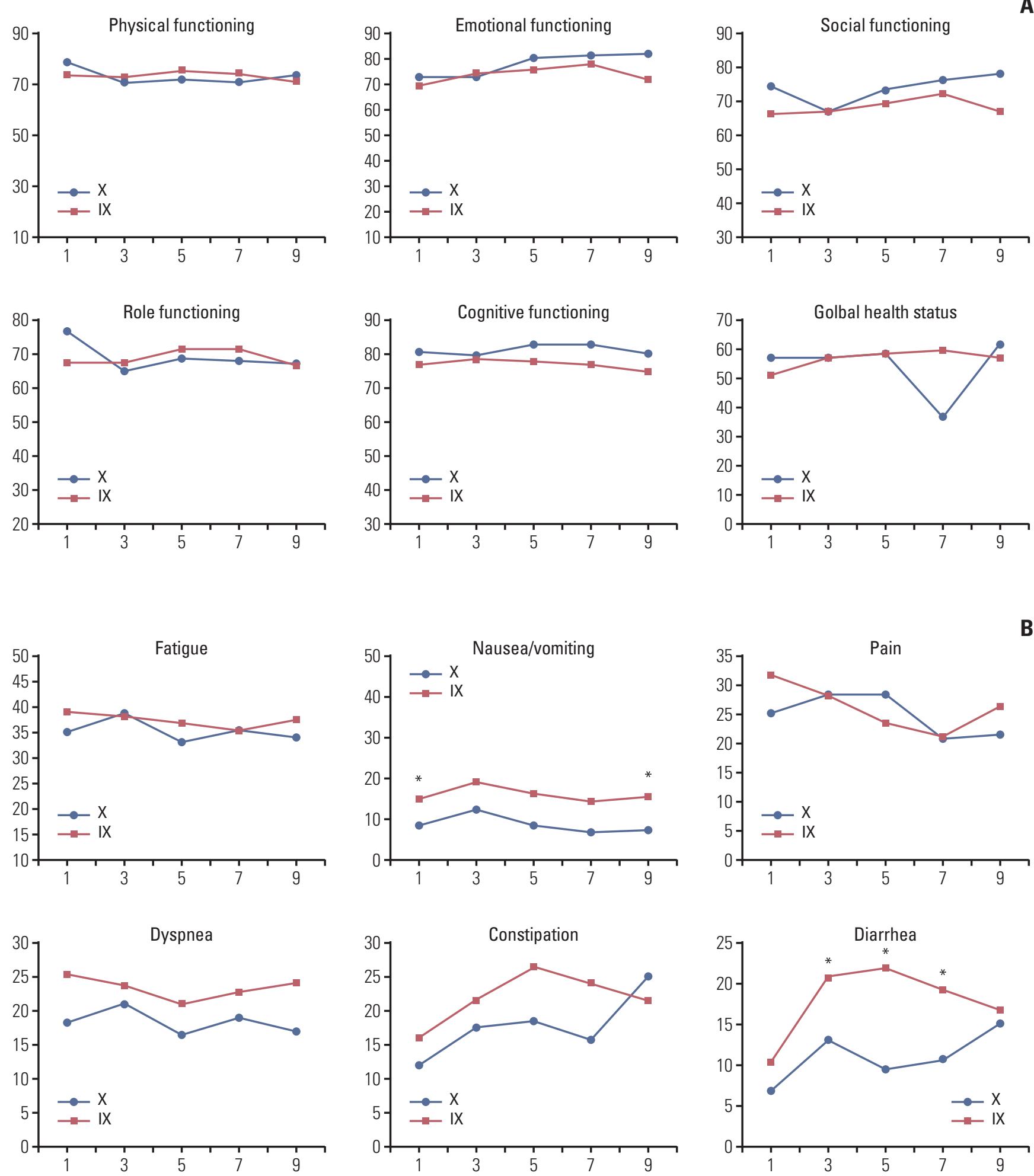

Fig. 5. Quality of life measurement. (A) The difference between baseline and each time point in global health and functional subscales. Positive values meant improved state compared with baseline. (B) The change in symptom subscales from baseline. In contrast to functional subscales, negative values meant improved state. IX, irinotecan and capecitabine combination; $X$, capecitabine. ${ }^{*} \mathrm{p}<0.05$. 
may have made it difficult to maintain adequate dose intensities of both irinotecan and capecitabine in this group.

Even though it was a subgroup analysis, results of the TNBC subgroup were intriguing. There have been few data regarding the benefits of combination therapy according to hormone receptor status. Similarly to our study, another phase III trial comparing ixabepilone plus capecitabine with single-agent capecitabine has demonstrated the superiority of combination treatment in a TNBC patient subgroup (HR, $0.64 ; 95 \%$ CI, 0.48 to 0.84 ) [18], whereas patients with hormone receptor-positive disease did not receive benefits from combination therapy (HR, 0.96 ; $95 \% \mathrm{CI}, 0.80$ to 1.14 ). Considering the more potent cytotoxic effects of combination therapy, it may be more reasonable to target hormone receptor negative-disease, which progresses rapidly with early drug resistance. Additionally, there was a tendency for OS improvement with irinotecan combination treatment in TNBC subgroup in this study, which was not adequately powered.

There were some concerns regarding safety of IX combination. Although most of the toxicities were manageable, neutropenia was the most pronounced toxicity that was responsible for frequent dose interruptions and delays. In addition, gastrointestinal symptoms such as diarrhea, nausea, and vomiting had significantly worse effects on QoL. In an earlier study, Perez et al. [7] has shown that weekly irinotecan is more tolerable and effective than every 3 weeks treatment. Although we did administer irinotecan on a weekly basis, the degree of toxicity seemed to be worse as combined with capecitabine.

Recently, the result of phase III clinical trial (BEACON) which compared oral irinotecan agent, etirinotecan pegol with treatment of physician's choice in patients with HER2 negative MBC was reported [19]. Etirinotecan pegol is designed to improve tissue distribution and reduce the toxicities of SN38, the active metabolite of irinotecan [20]. The toxicity profile of etirinotecan pegol was comparable to that of control arm, despite worse gastrointestinal symptoms ( $\geq$ grade 3 diarrhea $10 \%$ vs. $1 \%$ ) $[19,21]$. Although the study did not demonstrate an improvement in OS for etirinotecan pegol arm compared to control arm, subgroup analysis showed prolonged survival in patients with liver or brain metastasis, more aggressive disease [22]. Irinotecan may have promise for more challenging diseases such as TNBC or those with visceral metastasis, as inferred from BEACON trial and our study.

In summary, irinotecan and capecitabine combination did not prove clinically superior to capecitabine alone as treatment of HER2-negative anthracycline- and taxane-pretreated $\mathrm{MBC}$. Even though more AEs were found in combination treatment arm, most cases were tolerable and manageable. In view of the unmet need for effective drugs in highly refractory disease, irinotecan based chemotherapy could offer some benefits to patients with TNBC based on our subgroup analysis. Further clinical studies will be needed for irinotecan based treatment for this subgroup of patients. In addition, the reasonable approach to mitigate irinotecan toxicities should be planned as part of proactive supportive care.

\section{Conflicts of Interest}

This study was partly funded by Shin Poong Pharmaceuticals Co.

\section{Acknowledgments}

We thank the patients, their families, and all investigators who participated in the study. We also thank the Shin Poong pharmaceuticals Co. for providing Irinotecan.

This study was supported by NCC Grant No 1210530.

\section{Author Details}

${ }^{1}$ Division of Internal Medicine, Center for Breast Cancer, National Cancer Center, Goyang, ${ }^{2}$ Department of Internal Medicine, Seoul National University College of Medicine, Seoul, ${ }^{3}$ Department of Oncology, Asan Medical Center, Ulsan University College of Medicine, Seoul, 'Department of Oncology, Severance Hospital, Yonsei University College of Medicine, Seoul, ${ }^{5}$ Division of HematologyOncology, Department of Medicine, Samsung Medical Center, Sungkyunkwan University College of Medicine, Seoul, ${ }^{6}$ Division of Oncology / Hematology, Department of Internal Medicine, Korea University College of Medicine, Seoul, ${ }^{7}$ Division of Oncology/Hematology, Department of Internal Medicine, Seoul National University Bundang Hospital, Seongnam, ${ }^{8}$ Center for Clinical Trials, National Cancer Center, Goyang, ' Department of Internal Medicine, ChungAng University College of Medicine, Seoul, ${ }^{10}$ Department of Internal Medicine, Dong-A University College of Medicine, Busan, Korea 


\section{References}

1. Cardoso F, Costa A, Norton L, Senkus E, Aapro M, Andre F, et al. ESO-ESMO 2nd international consensus guidelines for advanced breast cancer (ABC2)dagger. Ann Oncol. 2014;25: 1871-88.

2. Wang JC. Cellular roles of DNA topoisomerases: a molecular perspective. Nat Rev Mol Cell Biol. 2002;3:430-40.

3. Pizzolato JF, Saltz LB. The camptothecins. Lancet. 2003;361: 2235-42.

4. Ichikawa W, Takahashi T, Suto K, Yamashita T, Nihei Z, Shirota $Y$, et al. Thymidylate synthase predictive power is overcome by irinotecan combination therapy with S-1 for gastric cancer. Br J Cancer. 2004;91:1245-50.

5. Guo Y, Shi M, Shen X, Yang C, Yang L, Zhang J. Capecitabine plus irinotecan versus 5-FU / leucovorin plus irinotecan in the treatment of colorectal cancer: a meta-analysis. Clin Colorectal Cancer. 2014;13:110-8.

6. O'Connor T, Rustum Y, Levine E, Creaven P. A phase I study of capecitabine and a modulatory dose of irinotecan in metastatic breast cancer. Cancer Chemother Pharmacol. 2008; 61:125-31.

7. Perez EA, Hillman DW, Mailliard JA, Ingle JN, Ryan JM, Fitch TR, et al. Randomized phase II study of two irinotecan schedules for patients with metastatic breast cancer refractory to an anthracycline, a taxane, or both. J Clin Oncol. 2004;22:2849-55.

8. Tan WW, Hillman DW, Salim M, Northfelt DW, Anderson DM, Stella PJ, et al. N0332 phase 2 trial of weekly irinotecan hydrochloride and docetaxel in refractory metastatic breast cancer: a North Central Cancer Treatment Group (NCCTG) Trial. Ann Oncol. 2010;21:493-7.

9. Stathopoulos GP, Tsavdaridis D, Malamos NA, Rigatos SK, Kosmas C, Pergantas N, et al. Irinotecan combined with docetaxel in pre-treated metastatic breast cancer patients: a phase II study. Cancer Chemother Pharmacol. 2005;56:487-91.

10. Lee KS, Park IH, Nam BH, Ro J. Phase II study of irinotecan plus capecitabine in anthracycline- and taxane- pretreated patients with metastatic breast cancer. Invest New Drugs. 2013;31:152-9.

11. Aaronson NK, Ahmedzai S, Bergman B, Bullinger M, Cull A, Duez NJ, et al. The European Organization for Research and Treatment of Cancer QLQ-C30: a quality-of-life instrument for use in international clinical trials in oncology. J Natl Cancer Inst. 1993;85:365-76.

12. Yun YH, Park YS, Lee ES, Bang SM, Heo DS, Park SY, et al. Validation of the Korean version of the EORTC QLQ-C30. Qual Life Res. 2004;13:863-8.

13. Jassem J, Carroll C, Ward SE, Simpson E, Hind D. The clinical efficacy of cytotoxic agents in locally advanced or metastatic breast cancer patients pretreated with an anthracycline and a taxane: a systematic review. Eur J Cancer. 2009;45:2749-58.

14. Gelmon K, Chan A, Harbeck N. The role of capecitabine in first-line treatment for patients with metastatic breast cancer. Oncologist. 2006;11 Suppl 1:42-51.

15. Belfiglio M, Fanizza C, Tinari N, Ficorella C, Iacobelli S, Natoli $\mathrm{C}$, et al. Meta-analysis of phase III trials of docetaxel alone or in combination with chemotherapy in metastatic breast cancer. J Cancer Res Clin Oncol. 2012;138:221-9.

16. O'Shaughnessy J, Miles D, Vukelja S, Moiseyenko V, Ayoub $\mathrm{JP}$, Cervantes G, et al. Superior survival with capecitabine plus docetaxel combination therapy in anthracycline-pretreated patients with advanced breast cancer: phase III trial results. J Clin Oncol. 2002;20:2812-23.

17. Albain KS, Nag SM, Calderillo-Ruiz G, Jordaan JP, Llombart AC, Pluzanska A, et al. Gemcitabine plus Paclitaxel versus Paclitaxel monotherapy in patients with metastatic breast cancer and prior anthracycline treatment. J Clin Oncol. 2008;26: 3950-7.

18. Sparano JA, Vrdoljak E, Rixe O, Xu B, Manikhas A, Medina C, et al. Randomized phase III trial of ixabepilone plus capecitabine versus capecitabine in patients with metastatic breast cancer previously treated with an anthracycline and a taxane. J Clin Oncol. 2010;28:3256-63.

19. Perez EA, Awada A, O'Shaughnessy J, Rugo HS, Twelves C, Im SA, et al. Etirinotecan pegol (NKTR-102) versus treatment of physician's choice in women with advanced breast cancer previously treated with an anthracycline, a taxane, and capecitabine (BEACON): a randomised, open-label, multicentre, phase 3 trial. Lancet Oncol. 2015;16:1556-68.

20. Hoch U, Staschen CM, Johnson RK, Eldon MA. Nonclinical pharmacokinetics and activity of etirinotecan pegol (NKTR102), a long-acting topoisomerase 1 inhibitor, in multiple cancer models. Cancer Chemother Pharmacol. 2014;74:1125-37.

21. Twelves C, Cortes J, O'Shaughnessy J, Awada A, Perez EA, Im SA, et al. Health-related quality of life in patients with locally recurrent or metastatic breast cancer treated with etirinotecan pegol versus treatment of physician's choice: results from the randomised phase III BEACON trial. Eur J Cancer. 2017;76: 205-15.

22. Cortes J, Rugo HS, Awada A, Twelves C, Perez EA, Im SA, et al. Prolonged survival in patients with breast cancer and a history of brain metastases: results of a preplanned subgroup analysis from the randomized phase III BEACON trial. Breast Cancer Res Treat. 2017;165:329-41. 